\title{
Serum cholinesterase: a potential assistant biomarker for hand, foot, and mouth disease caused by enterovirus 71 infection
}

\author{
Bang-Ning Cheng ${ }^{1 \dagger}$, Yu-Lian Jin ${ }^{2 \dagger}$, Bi-Quan Chen ${ }^{2}$, Li-Yan Zhu ${ }^{3}$, Zi-Cheng Xu and Tao Shen ${ }^{1,3^{*}}$
}

\begin{abstract}
Background: Hand, foot, and mouth disease (HFMD) caused by enterovirus 71 (EV71) is a potentially life-threatening infectious disease that commonly occurs in children. Diagnosis of HFMD caused by EV71 largely depends on clinical manifestations and rare serological biomarkers used to identify children suffering from HFMD. Serum cholinesterase (SChE) activity has frequently been reported as a potential biomarker for solid central nervous system tumors, chronic heart failure, and liver cirrhosis. However, its potential value in the diagnosis of neurotropic virus infections, such as HFMD caused by EV71, remains to be determined.
\end{abstract}

Findings: In our study, 220 children hospitalized with HFMD caused by EV71, 34 inpatients infected with coxsackievirus A16 (CVA16), and 43 undefined enterovirus-infected HFMD inpatients were recruited at the Anhui Provincial Children's Hospital between January 2011 and December 2012. SChE activity was measured. The non-parametric Mann-Whitney $U$ test showed that SChE activity in children diagnosed with HFMD caused by EV71 was significantly higher than in healthy controls $(p<0.001)$, as well as in children with upper respiratory tract infections $(p=0.011)$, bronchopneumonia $(p<0.001)$, septicemia $(p<0.001)$, amygdalitis $(p<0.001)$, and appendicitis $(p<0.001)$. In addition, higher SChE activity was observed in male inpatients with HFMD caused by EV71 (47.7\% positivity) compared to female inpatients (26.1\% positivity) (chi-square test, $p=0.002$ ). In our study, no significant differences in SChE levels were observed among different ages (up to 120 months) $(r=-0.112, p>0.05)$. An important finding was that SChE activity declined in the recovery phase of HFMD caused by EV71 compared to the acute phase $(p<0.001)$.

Conclusions: Elevated SChE activity was observed in patients with severe HFMD caused by EV71. Therefore, SChE might be a potential assistant biomarker for the diagnosis of HFMD caused by EV71 in children.

Keywords: Serum cholinesterase, Hand, foot, and mouth disease, Enterovirus 71, Coxsackievirus A16, Children, Biochemical marker

\footnotetext{
* Correspondence: taoshen@hsc.pku.edu.cn

${ }^{\dagger}$ Equal contributors

${ }^{1}$ Clinical Laboratory Center, Anhui Provincial Children's Hospital, Hefei

230051, China

${ }^{3}$ Department of Microbiology and Infectious Disease Center, School of Basic

Medical Sciences, Peking University, Beijing 100191, China

Full list of author information is available at the end of the article
} 


\section{Multilingual abstracts}

Please see Additional file 1 for translation of the abstract into the six official working languages of the United Nations.

\section{Findings}

Hand, foot, and mouth disease (HFMD) is a potentially life-threatening infectious disease commonly found in children. In China, 1,828,377 HFMD cases were diagnosed in 2013; among these, 252 cases resulted in death, according to the report from the national Health and Family Commission of China [1]. The main pathogens of HFMD are enteroviruses. In recent years, most cases of HFMD in mainland China were caused by enterovirus 71 (EV71) [2-4], followed by coxsackievirus A16 (CVA16), as well as other enterovirus serotypes. The diagnosis of HFMD caused by EV71 largely depends on the former's clinical manifestations, such as fever; vesicular rashes on palms, soles, and mouth; and, in severe cases, central nervous system (CNS) involvement $[3,5,6]$. Certain serological biomarkers are helpful for early diagnosis, timely surveillance, and appropriate medical treatment of HFMD, in order to avoid disease progression and death. Although some reports indicate that serum levels of cytokines, such as IL-2, IL-4, IL-10, IFN- $\gamma$, GM-CSF, and TNF- $\alpha$, may be independent risk factors for early medical intervention $[7,8]$, at present there are no reliable and easilyoperated biomarkers that could be used to assist diagnosis of HFMD in clinical practice.

Serum cholinesterase (SChE) is an enzyme synthesized by the liver. It has been reported that the enzyme's activity changes under certain disease conditions such as liver malfunction, obesity, diabetes, inflammation, and leptospiral infection [9, 10]. Serum cholinesterase is a routine parameter included in biochemical panels in clinical laboratory settings. Therefore, it could serve as a potential biomarker for clinical diagnosis. To date, characteristics of SChE activity have not been reported in HFMD, and its potential diagnostic value for HFMD is also unclear. Therefore in this study, we measured SChE activity in HFMD patients and evaluated its potential diagnostic value.

A total of 220 children hospitalized with HFMD caused by EV71, 34 inpatients infected with CVA16, and 43 inpatients infected with pan-enteroviruses (members of the enterovirus genus, PE) were recruited at the Anhui Provincial Children's Hospital between January 2011 and December 2012. All patients were aged one month to 10 years and exhibited complications, with varying degrees of CNS involvement, that caused symptoms such as myoclonus, ataxia, nystagmus, oculomotor palsies, and bulbar palsy in various combinations; and/or respiratory distress with tachycardia, tachypnea, and rales, with or without frothy sputum. All HFMD cases were diagnosed according to China's Ministry of Health guidelines [11]. Patient demographics, clinical symptoms, and major complications were collected retrospectively from the patients' medical histories. Whole blood samples from all participants were collected and used for routine blood testing and in serum biochemical parameter tests. Stool specimens were collected from children diagnosed with HFMD and kept at $-80{ }^{\circ} \mathrm{C}$ until examined for the presence of enteroviruses by reverse transcription polymerase chain reaction (RT-PCR). Viral RNA was extracted from the supernatant of $10 \%(\mathrm{~V} / \mathrm{V})$ stool specimens using the QIAamp Viral RNA Mini Kit (Qiagen, Hilden, Germany) and subjected to RT-PCR according to the manufacturer's instructions (DAAN Gene, Guangzhou, China) [12-14]. The RT-PCR kit specifically detected PE, EV71, and CVA16. The classification of EV71, CVA16, and PE depended on the analysis of realtime data. In addition, a total of 155 age-matched HFMD-negative inpatient children diagnosed with upper respiratory tract infections (URTIs) $(n=11)$, bronchopneumonia (BP) $(n=43)$, septicemia $(n=27)$, amygdalitis $(n=7)$, and appendicitis $(n=18)$, as well as 80 agematched healthy children, were used as controls. The study was approved by the institutional review board at Peking University Health Science Center and the Anhui Provincial Children's Hospital. Because the specimens were collected during the normal course of patient care, no informed consent was required, according to the Ethics Committee.

Serum samples were collected from clotted blood and detection of serum biochemical parameters was carried out within two hours of sample separation. Serum cholinesterase activity was determined using an assay, with butyrylthiocholine as the substrate. The analyses were done using Leadman reagent kits (Leadman, Beijing, China) on a Hitachi 7600 Automatic Biochemical Analyzer (Hitachi Ltd., Tokyo, Japan). The normal range of serum SChE was between 4500 and 13,000 IU/L, using the enzyme velocity method.

In our study, increased SChE activity, defined as higher than 13,000 IU/L, was observed in $40 \%$ of children with HFMD caused by EV71 (see Fig. 1a). The non-parametric Mann-Whitney $U$ test showed that SChE activity was significantly higher in children suffering from HFMD caused by EV71 than in healthy controls ( $0 \%$ positivity, $p<0.001)$, as well as children with URTIs (9.1\% positivity, $p=0.011)$, BP (16.3\% positivity, $p<0.001)$, septicemia (18.5\% positivity, $p<0.001)$, amygdalitis (0\% positivity, $p<0.001)$, and appendicitis ( $0 \%$ positivity, $p<0.001)$. No significant differences in SChE activity were observed among HFMD patients infected with EV71, CVA16 (35.3\% positivity), and $\mathrm{PE}(41.8 \%$ positivity). In 

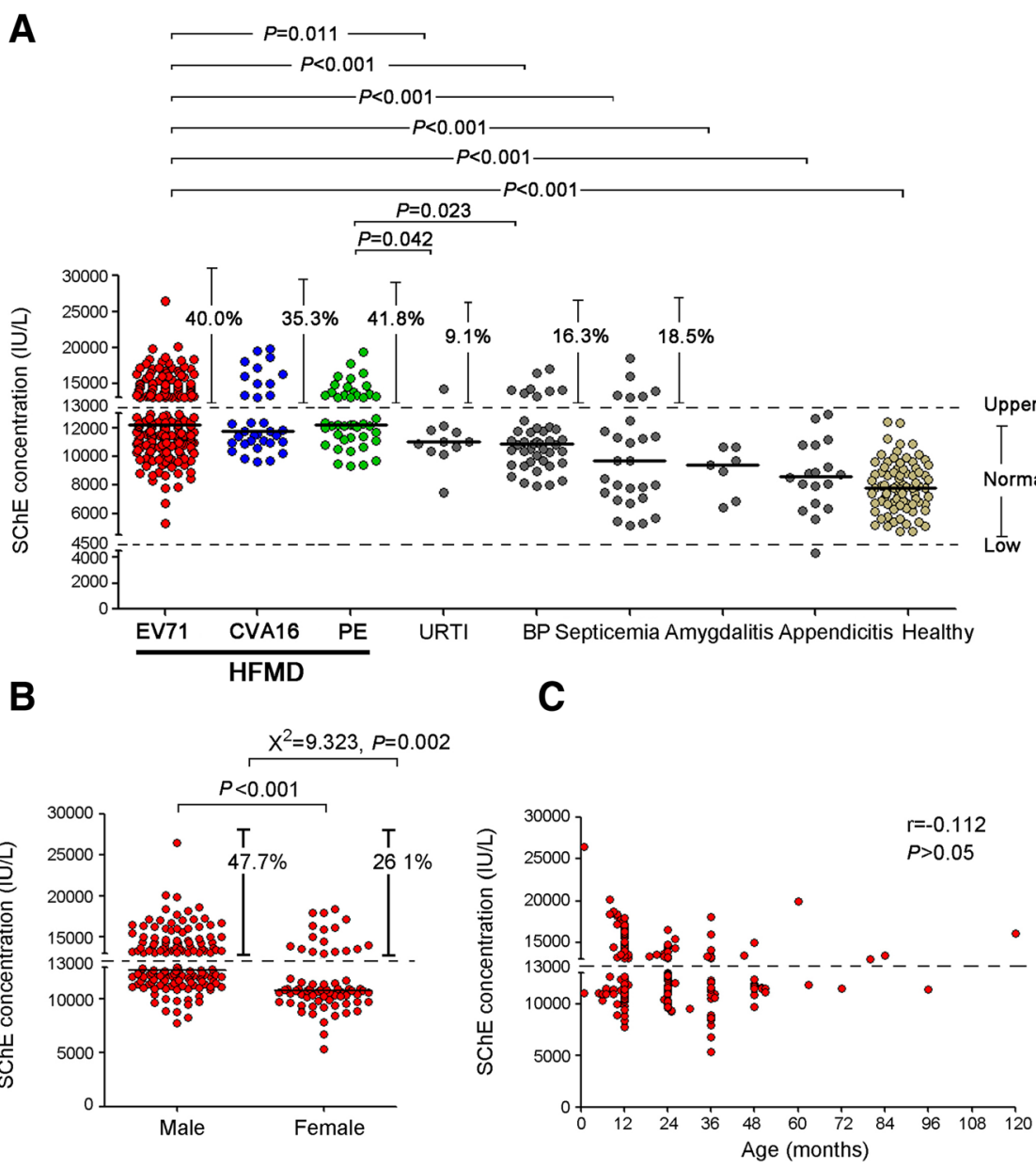

Fig. 1 Characteristics of SChE activity in inpatient children infected with HFMD caused by EV71. a SChE activity in 220 inpatient children infected with HFMD caused by EV71, 34 patients infected with CVA16, and 43 patients infected with PE was compared with SChE activity in healthy children $(n=80)$ and HFMD-negative children diagnosed with other common diseases including URTIs $(n=11)$, BP $(n=43)$, septicemia $(n=27)$, amygdalitis $(n=7)$, and appendicitis $(n=18)$. b Higher SChE activity was observed in inpatient males infected with HFMD caused by EV71 compared to female inpatients. c No significant differences were observed in elevated SChE levels among EV71-infected patients of different ages

addition, higher SChE activity was observed in male HFMD inpatients ( $47.7 \%$ positivity) compared to female inpatients (26.1\% positivity) (Student's $t$-test, $p<0.001$; chi-square test, $p=0.002$ ) (see Fig. 1b). Most cases of HFMD caused by EV71 occurred in children below the age of two, and particularly in infants under one year of age (see Fig. 1c). However, we did not observe a significant difference in elevated SChE levels among different ages (up to 120 months) $(r=-0.112, P>0.05$; see Fig. 1c). Furthermore, as shown in Fig. 2, after tracking SChE activity in 19 patients suffering from acute HFMD for more than two months, we found that serum SChE activity declined during the recovery phase compared to the acute phase of the disease $(p<0.001)$.

Cholinesterase consists of two members, i.e., acetylcholinesterase (AChE) and SChE [15]. Acetylcholinesterase is mainly found in erythrocytes, and in the central and peripheral nervous systems. Its main role is to catalyze the hydrolysis of the neurotransmitter acetylcholine into choline and acetic acid, a termination process necessary for neuronal transmission after activation. In contrast to AChE, SChE-also known as plasma cholinesterase, but yrylcholinesterase, or acetylcholine acylhydrolase-is synthesized primarily in the liver and distributed to circulating fluids. Serum cholinesterase serves mostly as a nonspecific esterase to cleave various esters, even those with bulkier acyl moieties [16]. Recently, SChE has been reported as a potential biomarker [17] for solid CNS tumors [18], chronic heart failure [19], liver cirrhosis [20, 21], parasympathetic dysfunction, and inflammation-related diseases [22], although the underlying mechanisms have yet not been clearly identified. To our knowledge, its potential value to diagnose neurotropic virus infections, such as EV71associated HFMD, is currently unclear. 


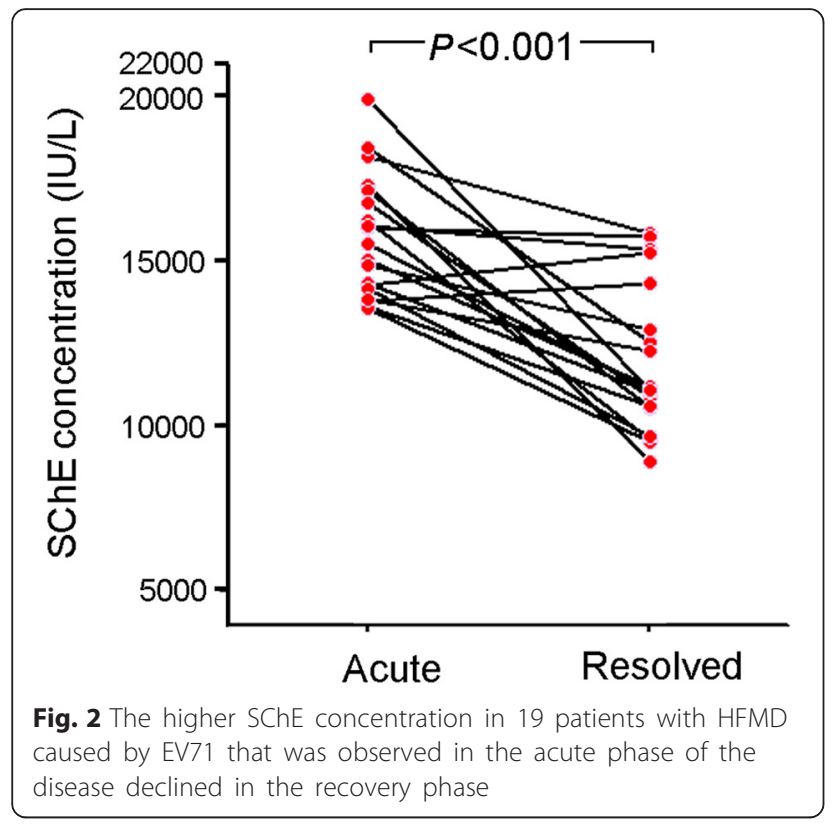

Our study had some limitations. Because the majority of the inpatient children suffering from HFMD that we studied were infected with EV71, our study failed to recruit a manageable number of patients infected with other enterovirus serotypes or other infectious diseases. Our study may indicate that higher SChE activity in HFMD inpatient children is not an EV71-specific phenomenon, but is also present in HFMD patients infected with other enteroviruses. This phenomenon should be verified in a study with a much larger cohort of non-EV71-infected HFMD patients. Also, it should be further analyzed if CNS symptoms complicated with HFMD caused by EV71 are associated with higher SChE activity.

Despite these limitations, our study is the first to report that SChE activity in peripheral blood is higher in HFMD children than in children diagnosed with other common diseases and healthy children. This clearly demonstrates that SChE activity can be used as a potential assistant biomarker for identifying children suffering from HFMD caused by EV71.

\section{Additional file}

Additional file 1: Multilingual abstracts in the six official working languages of the United Nations. (PDF 427 kb)

\section{Abbreviations}

AChE: acetylcholinesterase; BP: bronchopneumonia; CNS: central nervous system; CVA16: coxsackievirus A16; EV71: enterovirus 71; HFMD: hand, foot, and mouth disease; PE: enterovirus genus; RT-PCR: reverse transcriptase polymerase chain reaction; SChE: serum cholinesterase; URTI: upper respiratory tract infection.

\section{Competing interests}

The authors declare that they have no competing interests.

\section{Authors' contributions}

$\mathrm{TS}, \mathrm{BNC}$, and $\mathrm{YL}$ planned the study, and wrote the study plans and procedures. BQC, YLJ, and ZCX contributed to specimen collection. BNC and ZCX provided and supervised the laboratory testing for diagnosis. BNC, YLJ, LYZ, and ZCX undertook the data collection, management, and analysis. TS prepared the draft paper. All authors reviewed and approved the paper.

\section{Acknowledgments}

We thank all participants involved in this study. This study was supported by the National Natural Science Foundation of China (No: 81271826).

\section{Author details}

'Clinical Laboratory Center, Anhui Provincial Children's Hospital, Hefei 230051, China. ${ }^{2}$ Department of Infectious Diseases, Anhui Provincial Children's Hospital, Hefei 230051, China. ${ }^{3}$ Department of Microbiology and Infectious Disease Center, School of Basic Medical Sciences, Peking University, Beijing 100191, China.

Received: 9 September 2015 Accepted: 24 March 2016

Published online: 29 March 2016

\section{References}

1. Offical report of the National health and Family Planning Commission of China. http://www.nhfpc.gov.cn/jkj/s3578/201402/26700e8a83c04205913 a106545069a11.shtml. Accessed 13 Feb 2014.

2. Ooi MH, Wong SC, Lewthwaite P, Cardosa MJ, Solomon T. Clinical features, diagnosis, and management of enterovirus 71. Lancet Neurol. 2010;9(11): 1097-105.

3. Solomon T, Lewthwaite P, Perera D, Cardosa MJ, McMinn P, Ooi MH. Virology, epidemiology, pathogenesis, and control of enterovirus 71. Lancet Infect Dis. 2010;10(11):778-90.

4. Yang $\mathrm{T}, \mathrm{Xu} \mathrm{G}$, Dong $\mathrm{H}, \mathrm{Ye} \mathrm{M}, \mathrm{He} \mathrm{T}$. A case-control study of risk factors for severe hand-foot-mouth disease among children in Ningbo, China, 2010-2011. Eur J Pediatr. 2012;171(9):1359-64.

5. Huang CC, Liu CC, Chang YC, Chen CY, Wang ST, Yeh TF. Neurologic complications in children with enterovirus 71 infection. N Engl J Med. 1999:341(13):936-42.

6. Chang LY, Lin TY, Hsu KH, Huang YC, Lin KL, Hsueh C, et al. Clinical features and risk factors of pulmonary oedema after enterovirus-71-related hand, foot, and mouth disease. Lancet. 1999;354(9191):1682-6.

7. Li W, Teng G, Tong H, Jiao Y, Zhang $\mathrm{T}$, Chen $\mathrm{H}$, et al. Study on risk factors for severe hand, foot and mouth disease in China. PLoS ONE. 2014;9(1): e87603.

8. Wei R, Xu L, Zhang N, Zhu K, Yang J, Yang C, et al. Elevated antigen-specific Th2 type response is associated with the poor prognosis of hand, foot and mouth disease. Virus Res. 2013;177(1):62-5.

9. Chougule A, Hussain S, Agarwal DP. Prognostic and diagnostic value of serum pseudocholinesterase, serum aspartate transaminase, and serum alinine transaminase in malignancies treated by radiotherapy. J Cancer Res Ther. 2008:4(1):21-5.

10. da Silva CB, Wolkmer P, Da Silva AS, Paim FC, Tonin AA, Castro VS, et al. Cholinesterases as markers of the inflammatory process in rats infected with Leptospira interrogans serovar Icterohaemorrhagiae. J Med Microbiol. 2012; 61(Pt 2):278-84.

11. Ministry of Health of the People's Republic of China. Diagnosis and treatment guideline on hand-foot-mouth disease. Int J Res Mark. 2010; 30(24):1473-5.

12. He SJ, Han JF, Ding XX, Wang YD, Qin CF. Characterization of enterovirus 71 and coxsackievirus A16 isolated in hand, foot, and mouth disease patients in Guangdong, 2010. Int J Infect Dis. 2013;17(11):e1025-30.

13. Xing W, Liao Q, Viboud C, Zhang J, Sun J, Wu JT, et al. Hand, foot, and mouth disease in China, 2008-12: an epidemiological study. Lancet Infect Dis. 2014;14(4):308-18.

14. Liu B, Luo L, Yan S, Wen T, Bai W, Li H, et al. Clinical Features for Mild Hand, Foot and Mouth Disease in China. PLoS ONE. 2015;10(8):e0135503.

15. La Du BN, Lockridge O. Molecular biology of human serum cholinesterase Fed Proc. 1986;45(13):2965-9. 
16. Silman I, Sussman JL. Acetylcholinesterase: 'classical' and 'non-classical' functions and pharmacology. Curr Opin Pharmacol. 2005;5(3):293-302.

17. Santarpia L, Grandone I, Contaldo F, Pasanisi F. Butyrylcholinesterase as a prognostic marker: a review of the literature. J Cachex Sarcopenia Muscle. 2013;4(1):31-9

18. Mikecin L, Krizmaric M, Stepan Giljevic J, Gjurasin M, Kern J, Lenicek Krleza J, et al. Pseudocholinesterase activity in cerebrospinal fluid as a biomarker of solid central nervous system tumors in children. Croat Med J. 2013;54(5): 429-35.

19. Sato T, Yamauchi H, Suzuki S, Yoshihisa A, Yamaki T, Sugimoto K, et al. Serum cholinesterase is an important prognostic factor in chronic heart failure. Heart Vessels. 2015; 30(2): 204-210.

20. Meng F, Yin X, Ma X, Guo XD, Jin B, Li H. Assessment of the value of serum cholinesterase as a liver function test for cirrhotic patients. Biomed Rep. 2013;1(2):265-8.

21. Ramachandran J, Sajith KG, Priya S, Dutta AK, Balasubramanian KA. Serum cholinesterase is an excellent biomarker of liver cirrhosis. Trop Gastroenterol. 2014;35(1):15-20.

22. Shenhar-Tsarfaty S, Berliner S, Bornstein NM, Soreq H. Cholinesterases as biomarkers for parasympathetic dysfunction and inflammation-related disease. J Mol Neurosci. 2014;53(3):298-305.

\section{Submit your next manuscript to BioMed Central} and we will help you at every step:

- We accept pre-submission inquiries

- Our selector tool helps you to find the most relevant journal

- We provide round the clock customer support

- Convenient online submission

- Thorough peer review

- Inclusion in PubMed and all major indexing services

- Maximum visibility for your research

Submit your manuscript at www.biomedcentral.com/submit 